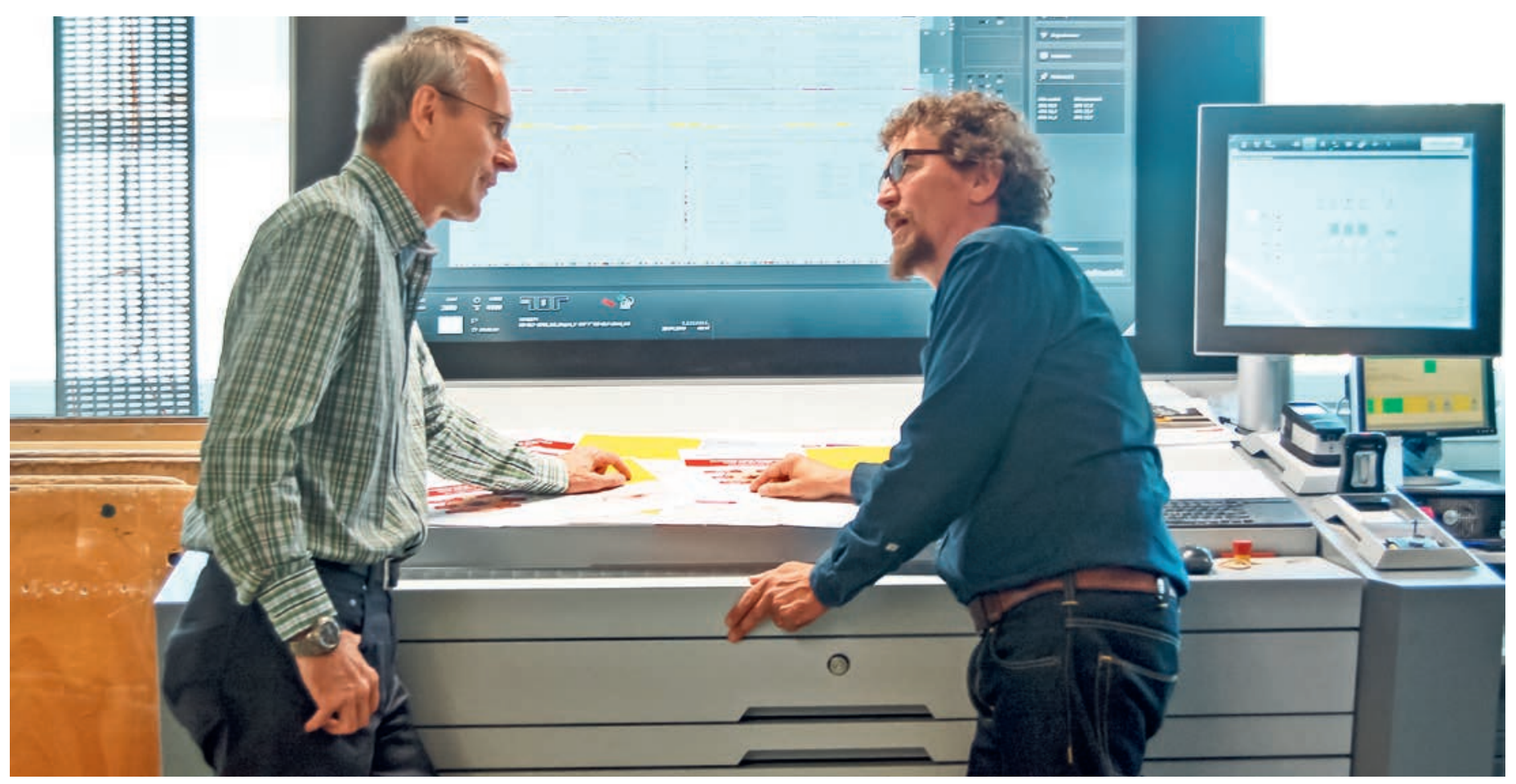

Bruno Kesseli (links) führt Matthias Scholer schrittweise in die Aufgaben des Chefredaktors ein.

\title{
Wechsel des Chefredaktors bei der Schweizerischen Ärztezeitung
}

Hans Kurt

Dr. med., Präsident des Verwaltungsrats EMH

Nach langjähriger Kontinuität kommt in diesem Sommer Bewegung in die Führungsetage der Schweizerischen Ärztezeitung. Der aktuelle Chefredaktor Bruno Kesseli verlässt die SÄZ und den Schweizerischen Ärzteverlag EMH per Ende Juli, weil er sich nach rund 14 Jahren Redaktionstätigkeit wieder vollumfänglich der praktischen ärztlichen Arbeit widmen möchte. Sein Amt übernimmt mit Matthias Scholer ein ebenso erfahrener wie breit ausgebildeter Journalist, der bereits seit einigen Jahren als Print- und Online-Redaktor für die SÄZ tätig ist.

Der Verwaltungsrat des Schweizerischen Ärzteverlags EMH hat Matthias Scholer zum neuen Chefredaktor der Schweizerischen Ärztezeitung (SÄZ) gewählt. Das oberste Organ von EMH stufte es wie auch der Zentralvorstand der FMH als Glücksfall ein, dass mit Matthias Scholer ein hochqualifizierter interner Kandidat für das wichtige Amt des SÄZ-Chefredaktors zur Verfügung stand.
Matthias Scholer verfügt über einen langjährigen Hintergrund im wissenschaftlichen und gesundheitspolitischen Journalismus. Von besonderem Gewicht ist in einer Zeit rascher medialer Entwicklungen seine publizistische Vielseitigkeit. Matthias Scholer ist im Printjournalismus ebenso versiert wie im Online- und Social-Media-Bereich. Zudem verfügt er über vertiefte Erfahrungen als Videojournalist. Bei der SÄZ ist er seit 
einigen Jahren für den Aufbau der crossmedialen Kommunikation und die Weiterentwicklung des OnlineAngebots der SÄZ sowie die Betreuung der Website und der Social-Media-Kanäle zuständig. Daneben verfasst er regelmässig Beiträge für die Printausgabe und gibt auf konzeptioneller Ebene wichtige Impulse für die Weiterentwicklung der Zeitschrift.

\section{Matthias Scholer verfügt über einen langjähri- gen Hintergrund im wissenschaftlichen und gesundheitspolitischen Journalismus.}

Nach Abschluss seiner Ausbildung zum Veterinärmediziner und der Promotion sowie einigen Jahren Tätigkeit im erlernten Beruf fasste Matthias Scholer rasch im Wissenschaftsjournalismus Fuss. Neben Arbeiten für grosse Schweizer Zeitungen wie NZZ und Tages-Anzeiger war er unter anderem als Medizinischer Redaktor für die Maurice E. Müller Foundation, als Redaktor und Managing Editor der Zeitschrift Care Management, als Kommunikationsverantwortlicher von SystemsX.ch, einer Geschäftsstelle der ETH Zürich, und als Projektleiter für den Schweizerischen Ärzteverlag EMH tätig. Daneben betrieb er mit SciMed eine eigene Agentur für Wissenschaftsjournalismus und leitete an der Schweizer Journalistenschule maz den CAS Fachjournalismus. Die Übernahme der Stelle als Chefredaktor der SÄZ kann vor diesem Hintergrund als logischer Schritt gesehen werden.

\section{Ab August Vollzeit in der Hausarztpraxis}

Der aktuelle Chefredaktor Bruno Kesseli, Facharzt für Allgemeine Innere Medizin und Journalist mit geisteswissenschaftlichem Hochschulabschluss, führt die SÄZRedaktion mittlerweile seit über 13 Jahren. Zu seinen ersten Projekten zählten eine umfassende strukturelle und gestalterische Überarbeitung des Zeitschriftenkonzepts sowie der Aufbau einer eigenständigen Redaktion. Daraus ging das «duale Konzept» der SÄZ hervor. Es ist gekennzeichnet durch einen klar als solchen erkennbaren offiziellen Teil, den «FMH-Teil» der SÄZ, sowie einen «freien», nach journalistischen Kriterien konzipierten
Teil. Für Letzteren zeichnet eine im Rahmen des Redaktionsstatuts unabhängige Redaktion verantwortlich. Auch in wirtschaftlich zunehmend schwierigen Zeiten gelang es der Redaktion unter der Leitung von Bruno Kesseli, mit beschränkten Ressourcen die Zeitschrift weiterzuentwickeln und deren journalistische Qualität zu steigern. Den Herausforderungen des digitalen Zeitalters konnte sich die SÄZ erfolgreich stellen, nicht zuletzt dank der Verstärkung des Redaktionsteams durch Matthias Scholer.

Bruno Kesseli setzte auch in seiner persönlichen beruflichen Ausrichtung auf ein «duales Konzept»: Neben seinem Engagement für die SÄZ war er in Teilzeit als Hausarzt aktiv. In den letzten zwei Jahren wuchs er stärker in die hausärztliche Arbeit hinein, so dass der Wunsch aufkam, sich nach knapp vierzehn spannenden und erfüllten Jahren bei der SÄZ nochmals in einem Vollzeitpensum der praktischen ärztlichen Tätigkeit zu widmen.

\section{Der Verwaltungsrat dankt Bruno Kesseli für seine sorgfältige Arbeit, für die kollegiale Zusammenarbeit und seine Integrität.}

Der Verwaltungsrat dankt Bruno Kesseli für seine sorgfältige Arbeit, für die kollegiale Zusammenarbeit und seine Integrität. Er war ein wertvolles und von allen akzeptiertes Bindeglied zwischen den FMH-Mitgliedern und dem EMH Verlag. Dem neuen Chefredaktor Matthias Scholer wünscht der Verwaltungsrat viel Erfolg in der Umsetzung seines reichen Ideenschatzes sowie die dazu nötige Akzeptanz und Anerkennung in der Ärzteschaft.

Ein Interview mit Bruno Kesseli folgt in den nächsten Wochen. Für den Monat Juli ist je ein «Zu guter Letzt» des scheidenden sowie des designierten Chefredaktors vorgesehen.

Bildnachweis

Elisa Jaun 